\title{
Intradiploic meningioma- A rare osteolytic calvarial lesion: A case report and review of literature
}

\author{
Sandeep B V', Kaushik Roy², Manpreet Singh Banga', Partha Ghosh' \\ ${ }^{1}$ Post Doctoral Trainee, ${ }^{2}$ Associate Professor, Department of Neurosurgery, Nilratan Sircar Medical College and \\ Hospital, Kolkata, West Bengal, India
}

\section{A B S T R A C T}

Meningiomas are most common benign intracranial neoplasm which is encountered in clinical practice. One of the most uncommon variant is intradiploic meningiomas, which can present as osteoblastic or osteolytic calvarial lesion and should be considered as differential diagnosis. We here present a rare case report of osteolytic intradiploic meningioma of skull presenting as scalp mass with a background of head injury and discuss its management.

Key words: Intradiploic meningioma, osteolytic lesion, calvarial lesion
Access this article online

Website:

http://nepjol.info/index.php/AJMS

DOI: 10.3126/ajms.v7i6.15157

E-ISSN: 2091-0576

P-ISSN: 2467-9100

\section{INTRODUCTION}

The meningiomas that originate in the skull have been referred to as calvarial, intraosseus or intradiploic. ${ }^{1}$ Winkler, in 1904, first described a meningioma originating in an extradural location. ${ }^{2}$ There are different case reports regarding intraosseous intradiploic meningiomas although it is a rare entity. These tumours are mainly osteoblastic and few cases reported are osteolytic in nature. ${ }^{3}$ Of these osteolytic type is more malignant than osteoblastic type of meningioma. Intradiploic meningiomas should be considered in differential diagnosis of osteolytic or osteoblastic skull lesions. ${ }^{1}$

\section{CASE REPORT}

A 53 year old female patient referred a slowly growing lump in right frontal region since one year six months which was painful. She related her complaints to trivial head injury occurred at that time.

On examination there was a single $3 \times 4 \mathrm{~cm}$ palpable bony lesion in right frontal region which was hard in consistency, non mobile, tender on palpation and skin was not adherent to the mass. The neurological examination showed no abnormalities. Chest X-ray and other laboratory investigations were normal. CT brain showed a osteolytic hypodense lesion in right frontal bone with erosion of both tables of skull and indentation of brain parenchyma (Figure 1).

On MRI brain there was a well circumscribed extraaxial lesion in right frontal region involving right frontal bone measuring $36.5 \times 29.5 \times 27.5 \mathrm{~mm}$ which was hypo to isotense on $\mathrm{T} 1 \mathrm{w}, \mathrm{T} 2 \mathrm{w}$ and flair images and shows heterogenous enhancement on post contrast study, In one part it seems to invade the dura but not involving the brain parenchyma (Figure 2).

Surgical exploration through right fronto parietal craniotomy encircling the mass was planned. Scalp and pericranium was free, outer table over the mass was thinned out and become egg shell type. The mass was totally extradural and came out with free bone flap (Figure 3).

There was dural involvement with inner portion of the lesion, which was removed along the tumor. Tumor was firm with grating consistency moderately vascular. Duroplasty followed by cranioplasty was done following excision of the mass.

\section{Address for correspondence:}

Dr. Sandeep B V, Department of Neurosurgery, $6^{\text {th }}$ floor, U.N.B. Building, Nilratan Sircar Medical College \& Hospital,

138, A J C Bose Road, Sealdah, Kolkata, West Bengal - 700 014, India.

E-mail: sandybv200@gmail.com, Phone: +919051206951 
Post operative events were uneventful and patient was discharged on $8^{\text {th }}$ post operative day. Histopathological examination of the lesion showed transitional cell meningioma (WHO grade 1) (Figure 4).

\section{DISCUSSION}

Meningiomas occur with an incidence of 2.1 per 100,000 cases. They appear in patients 20 to 60 years of age with a female-to-male ratio of approximately $2: 1{ }^{4}$ Intraosseous meningiomas constitute only $2 \%$ of meningiomas. ${ }^{5}$

'Primary intraosseous meningioma' is a subset of extradural meningiomas that arise in bone. ${ }^{6}$ True primary intraosseous meningiomas do not involve the dura. To avoid confusion, Lang et al. classified interosseous meningiomas as purely extracalvarial (type I), purely calvarial (type II), or calvarial with extracalvarial extension (III). The latter two are further

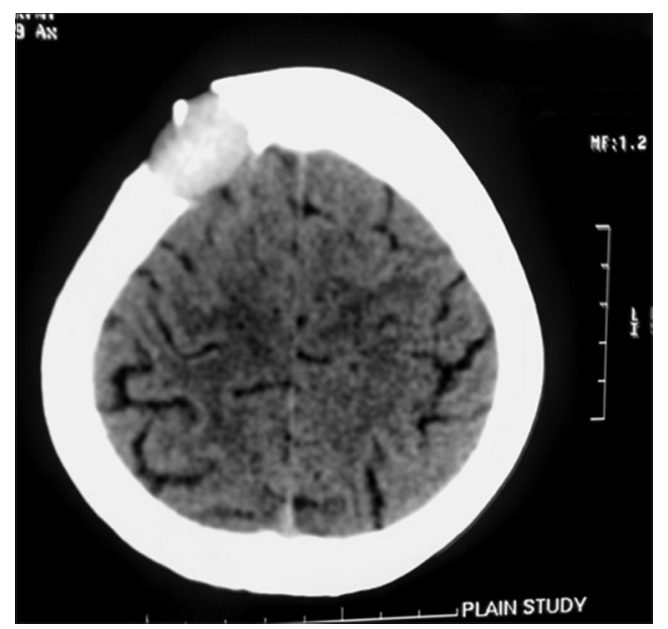

Figure 1: CT scan brain showing right frontal intradiploic osteolytic lesion

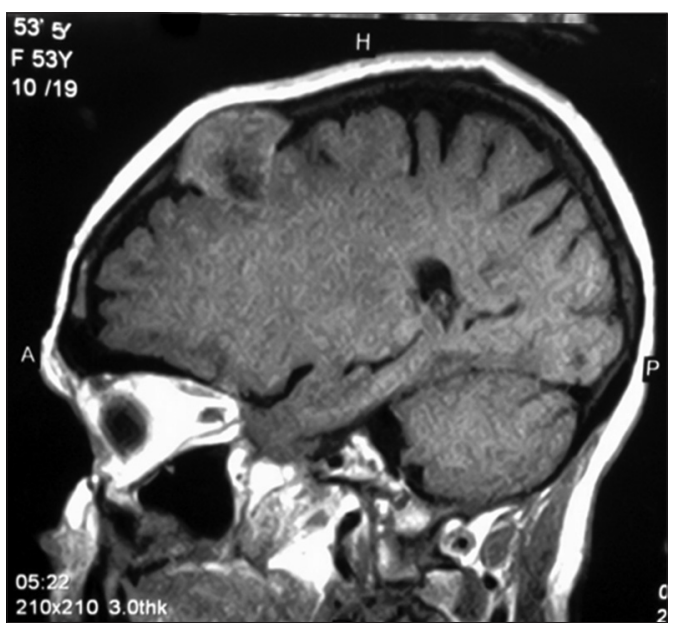

Figure 2: T1 MRI brain saggital cuts showing frontal extra axial heterogenous lesion abutting the brain parenchyma divided into convexity (C) or skull base (B) forms. ${ }^{7}$ Our case seems to be type III C of Langs classification.

Convexity and the skull base are the two major locations for occurrence of intraosseous meningiomas and average age of occurrence is 50.5 years with slight female preponderance. ${ }^{8}$

The origin of these tumors is controversial. Some believe they originate from arachnoid cap cells from normal dura and in the arachnoid granulations. Others postulate that they can arise from ectopic meningocytes which are trapped in the cranial sutures during molding of the head at the birth or following blunt trauma. ${ }^{9}$ A few recently reported cases had a history of trauma to the head and a subsequent fracture line near the region of the intraosseous meningioma. ${ }^{10}$ In our patient there was history of trauma to head was present but there was no fracture line detected.

The initial symptom of the patient is usually a painless expansile mass with normal neurological findings. The

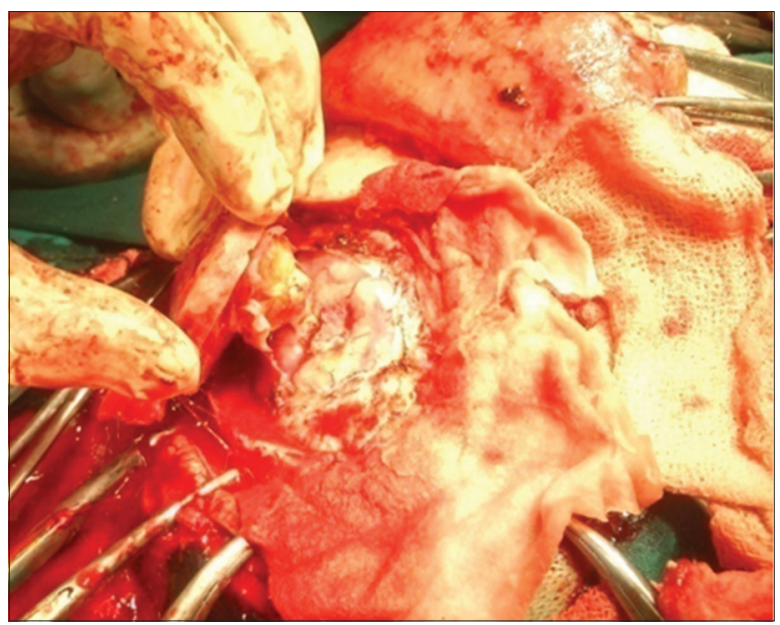

Figure 3: Excision of tumour with involved bone and dura

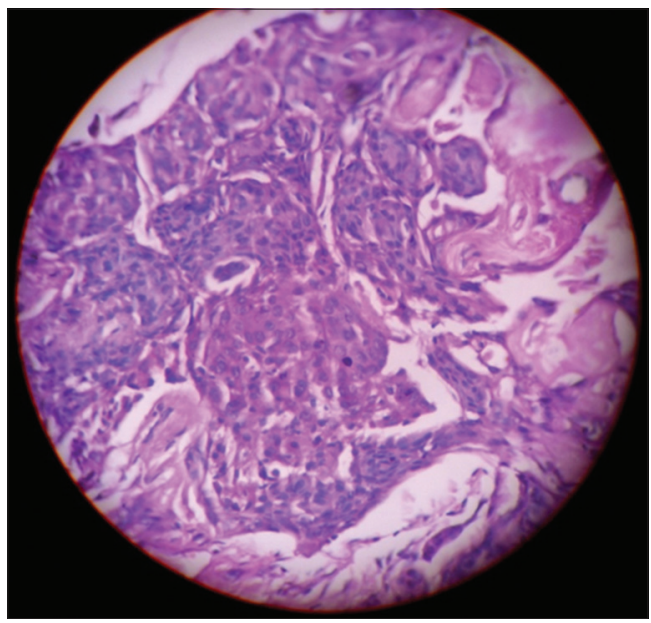

Figure 4: Histopathology slide showing presence of psammoma bodies suggestive of transitional cell meningioma

Asian Journal of Medical Sciences | Nov-Dec 2016 | Vol 7 | Issue 6 
symptoms usually depend on tumor size, location and involvement of the surrounding structures. ${ }^{11}$ In our case there was no neurological features attributed to the mass.

Meningiomas presenting with scalp swelling and extracranial soft-tissue masses are usually more aggressive than others in nature. ${ }^{11}$ Osteolytic meningiomas associated with softtissue component should be considered malignant until otherwise proven. ${ }^{12}$ In our case there was osteolytic bone lesion but the soft tissues of the scalp was not involved and histologically benign one (WHO grade 1).

Radiographic findings of intradiploic meningiomas are limited and are not pathognomonic because of the superimposed bony structures. The tumors are usually either osteoblastic or osteolytic lesions, although combined versions have been reported. Plain X-ray skull film can detect abnormalities, usually in the osteoblastic type. Hyperostosis, irregular calcification, and atypical vascular marking may be seen in some cases. The osteolytic type can be seen as hypodense appearance on plain radiographs. CT Brain shows a contrast enhancing isodense lesion. ${ }^{13}$ In our case $\mathrm{X}$ ray skull was not done. CT brain showed a osteolytic hypodense lesion in right frontal bone with erosion of both tables of skull and indentation of brain parenchyma.

MRI T1-weighted images show isointense or hypointense lesions compared with the brain while T2-weighted images are variable but usually hyperintense however hypointensity does not exclude the diagnosis of meningioma. Prominent homogeneous enhancement after Gadolinium (Gd) administration is seen typically. Normally the lesions do not show 'dural tail'. If the dural tail is seen, it could be secondary to dural invasion or irritation by the tumor. ${ }^{14}$ In our case, the lesion was was hypo to isotense on T1w, T2w and flair images and shows heterogenous enhancement on post contrast study without dural tail appearance.

Histopathologically, these lesions may appear from epithelial to mesenchymal in origin like their intracranial menigiomas. ${ }^{13}$ In our case histopathological examination revealed transitional cell meningioma (WHO grade 1 lesion).

The only curative treatment modality is total resection. Craniaplasty should be done if the surgical resection is wide as done in our case. When the tumor is resected subtotally because of the involvement of other critical structures, the residual tumor should be followed up imaging and radiation therapy should be considered..$^{10}$ Post operative imaging suggested no residual tumour in our case.

\section{CONCLUSION}

Although rare, osteolytic primary intraosseous meningioma should be considered as one of the differential diagnosis in evaluating calvarial lesions and wide excision is always preferable to avoid complications and achieve total cure.

\section{REFERENCES}

1. Sambasivan $M$, Sanalkumar $P$ and Mahesh S. Primary Intradiploic Meningioma. Kerala Medical Journal 2011;4:16-18.

2. PompiliA, Caroli $F$, Cattani F and lachetti M. Intradiploic meningioma of the orbital roof. Neurosurgery Journal 1983;12:565-568.

3. Monteiro JT, Baptista AE, Trabulo AS and De Almeida C. Intradiploic meningioma of the skull: case report and review of the literature. Neurocirugía 1996; 7:129-132.

4. Changhong L, Naiyin C, Yuehuan G and Lianzhong Z. Primary intraosseous meningiomas of the skull. Clinical Radioliology 1997; 52:546-549.

5. Muzumdar DP, Vengsarkar US, Bhatjiwale MG and Goel A. Diffuse calvarial meningioma: a case report. Journal of Postgraduate Medicine 2001; 47:116-118.

6. Maria P, Bernd FMR, Panagiotis P, Abdullah N, Tobias S, Wolfgang $F$, et al. Intraosseous hemangioma of the skull with dural tail sign: Radiologic features with pathologic correlation. American Journal of Neuroradiology 2005; 26:2049-2052.

7. Lang FF, Macdonald OK, Fuller GN and Demonte F. Primary extradural meningiomas: a report on nine cases and review. The literature from the era of computerized tomography scanning. Journal of Neurosurgery 2000; 93:940-950.

8. McGuire TP, Palme CE, Perez-Ordonez B, Gilbert RW and Sandor GK. Primary intraosseous meningioma of the calvaria:. analysis of the literature and case report. Oral Surg Oral Med Oral Pathol Oral Radiol Endod 2007; 104:34-41.

9. Azar-Kia B, Sarwar M, Marc JA and Schechter MM. Intraosseous meningioma. Neuroradiology 1974; 6:246-253.

10. Crawford TS, Kleinschmidt-DeMasters BK and Lillehei KO. Primary intraosseous meningioma. Case report. Journal of Neurosurgery 1995; 83:912-915.

11. Muthukumar N. Primary calvarial meningiomas. British journal of Neurosurgery 1997; 11:388-392.

12. Partington MD, Scheithauer BW and Piepgras DG. Carcinoembryonic antigen production associated with an osteolytic meningioma. Case report. Journal of Neurosurgery 1995; 82:489-492.

13. Mathur $\mathrm{N}$ and Borale $\mathrm{S}$. MRI findings of a very rare case of Intradiploic Meningioma. Bombay Hospital journal 2010; 52:444-446.

14. Arana E, Menor F and Lloret RM. Intraosseous meningioma. Journal of Neurosurgery 1996;85:362-363.

\footnotetext{
Authors Contribution:

SBV - Principle author, reviewed the literature, manuscript preparation and critical revision of the manuscript; KR - Operated the patient, reviewed the literature, manuscript preparation and critical revision of the manuscript; MSB - Literature search, prepared first draft of manuscript and critical revision of the manuscript; PG - Collected data and review of study, literature search.

Source of Support: Nil. Conflict of Interest: None.
} 